\title{
The geometry of classical Regge calculus
}

\author{
J W Barrett \\ Institute of Theoretical Physics, University of Zurich, Schoenberggasse 9, 8001 Zurich, \\ Switzerland
}

Received 19 November 1986, in final form 11 May 1987

\begin{abstract}
In the first section of this paper, standard notions of Riemannian geometry are applied to the case of piecewise-flat manifolds. Particular care is taken to explain how one may define some particular vectors and tensors in an invariant way at points of a conical singularity.

The geometry surrounding the equations of motion and the energy-momentum of the piecewise-flat manifold is developed in detail. The resolution theorem is presented, which states that on certain resolution hypersurfaces there is a clear connection between the energy-momentum of the piecewise-flat manifold and the Regge equations of motion. This theorem both generalises and explains a previous result of the author.
\end{abstract}

\section{Introduction}

This paper forms part of an investigation into the 'classical convergence problem' of Regge calculus. The problem is essentially to find, if such exists, a useful formulation of convergence for piecewise-flat manifolds which are solutions of Regge's equations (also called Regge manifolds or Regge skeleton spaces).

One really wishes to answer the following question: do solutions of Regge's equations really approximate solutions of Einstein's equations? This is rather an imprecise question, and to replace it with something more precise we can ask for a formulation of convergence of Regge solutions to smooth metrics.

Since we are dealing with solutions of the Regge equations, it becomes crucial to relate the Regge equations to geometrical properties of the piecewise-flat manifold. This is the nature of the results in this paper. The geometrical property in question is the flow of energy-momentum through a hypersurface of a piecewise-flat manifold. The relationship between this and the Regge equations of motion is called the resolution theorem.

The paper starts in $\$ 2$ with a rather general development of some aspects of the geometry of piecewise-flat manifolds. An account is given of the particular way in which standard notions of Riemannian geometry can be applied to piecewise-flat manifolds. Section 3 defines the energy-momentum in the piecewise-flat case.

The resolution theorem is presented in $\$ \S 4$ and 5 . This result was previously described in a short paper for the special case of the Einstein term in four dimensions (Barrett 1985, 1986). Some results in a similar direction appeared independently in a paper by Miller (1986). At that time, the reason why the calculation succeeded seemed somewhat mysterious. The general method of this paper provides some explanation, as well as extending the scope of the results to include a cosmological term, and to work in any dimension. 
In $N$ dimensions, the existence of $(N-1)$-dimensional resolution hypersurfaces is demonstrated for which, in a particular sense, the energy-momentum flow is precisely zero. Now clearly the energy-momentum cannot be zero pointwise on a Regge manifold (as is obvious from the discussion of $\S 3$ ). The sense in which the energy-momentum flow can be considered zero is the following. The energy-momentum is integrated 'piecewise' on the hypersurface, i.e. one splits the hypersurface into a number of cells, performing the integration on each cell, and having as a result an energy-momentum vector in the tangent space at each cell. These vectors are resolved into a sum of components, and then these components are re-summed in different sets, in a particular way dictated by the simplicial structure of the manifold. The resulting quantities are zero in the case that the Regge equations are satisfied. We might say that neighbouring contributions to the energy-momentum flow cancel. This is a slightly tricky point, as one cannot sum all the contributions to form a 'total energy-momentum', because of the curved nature of the manifold.

The resolution hypersurfaces are (the polyhedra of) particular subcomplexes of the derived complex of the manifold. Sections 4 and 5 include a proof that these subcomplexes actually form a manifold, and describe how they correspond to partitions of the manifold. Roughly speaking, for any way in which a hypersurface can cut the vertices of each simplex into two sets, one on each side of the surface, there exists a resolution hypersurface (we have in mind hypersurfaces which do not wrap themselves around vertices in any topologically complicated ways).

Other papers have appeared on the somewhat different problem of the convergence of the action, for different types of sequences than the ones which we would wish to consider, those related to a smooth metric by a geodesic triangulation (Cheeger et al 1984) or those related to a smooth metric via some embedding in a flat space of a high dimension (Feinberg et al 1984).

A good reference for general aspects of simplicial topology is the book by Maunder (1980).

In this paper only positive metrics are considered.

\section{The differential geometry of a Regge manifold}

The idea of this section is not so much to be an introduction to Regge calculus itself (Regge 1961), but to introduce the methods of differential geometry which are applied to Regge manifolds.

If, in $N$ dimensions, the $(N-2)$-simplexes (called the hinges, the places where the conical singularities are), are removed, the resulting manifold is an ordinary smooth differentiable manifold, and so we can use all the standard techniques of differential geometry-tensor fields, covariant derivatives and so on. A simplification occurs because the curvature vanishes here, as shall be described below. This is complemented by an account of the geometry at the singularities. What vectors or tensors can one define at a point of a conical singularity, and how do these relate to tensor fields on the flat part of the manifold?

\subsection{The simplex}

Let us start with a simplex. An $N$-simplex is an affine space, which means that, although it is defined as a portion of $R^{N}$, the location of the origin is unimportant. 
We think of displacement vectors, the difference of two points in the simplex, as vectors in $R^{N}$ however. In this sense $R^{N}$ is the tangent space of the simplex.

The simplex may also be viewed as a differentiable manifold, with a tangent space at each point, and with a flat (zero curvature) connection connecting the tangent spaces at different points. Of particular interest to us are the tensor fields which are constant on the simplex, i.e. ones with zero covariant derivative, and the tensor fields which vary linearly over the simplex, meaning ones whose covariant derivative is a constant tensor field. Notice that the space of constant vector fields is just $R^{N}$ and can be conveniently identified with what we previously called the tangent space of the simplex.

All affine $N$-simplexes are isomorphic. This no longer remains the case if we introduce a metric on the tangent space. Using the metric we can evaluate the squared lengths of the edges (the term edge will always mean a 1-simplex), since their displacement vectors are elements of the tangent space. Conversely, given a set of the $\frac{1}{2} N(N+1)$ edge lengths squared, one can uniquely determine the metric, which also has $\frac{1}{2} N(N+1)$ independent components. Thus the space of metric simplexes is $R^{N(N+1) / 2}$. The space can be divided into different parts, corresponding to the different possible signatures for the metric. From now on, we shall consider only the Euclidean metrics, and so the edge lengths will satisfy certain 'triangle' inequalities (Hartle 1985).

\subsection{Two simplexes}

Now we turn our attention to the problem of glueing together two metric simplexes. This is done so that the metrics on the common face agree, or equivalently, so that the edges in the common face have a unique length, regardless of which simplex they are regarded as belonging to. This means that later, when we come to consider manifolds made by glueing together many simplexes, the space of metrics on the manifold is equivalent to the space of lengths of the edges in the manifold (which are restricted by the above-mentioned inequalities).

To complete the glueing, we have to consider the affine connection. The problem is to specify an identification of the two tangent spaces, so that tangent vectors can be parallelly transported across from simplex to simplex or, thinking of these simplexes as Riemannian manifolds, to specify a glueing of the two tangent bundles together. The Christoffel choice of connection is to decompose a tangent vector in one simplex as a vector in the plane of the common face plus a multiple of the unit normal to the face. Then, on the other side of the fence, we can reconstruct the vector as the same vector in the face (because the face can be regarded as lying in either simplex), plus the same multiple of the unit normal vector (in the new simplex) to the face (the normals both pointing appropriately the same way, i.e. one outwards and one inwards).

Notice that, with this connection, the manifold of the two glued simplexes is completely flat and is isomorphic to a piece of $R^{N}$ with the standard Euclidean metric and connection (shaped like two simplexes stuck together!).

\subsection{Torsion}

A more general connection can be obtained by considering any orthogonal rotation between the two tangent spaces. If the connection is not the Christoffel one, however, there will be a distributional torsion field on the common face (Drummond (1986); there this is referred to as interface torsion). There are two ways of seeing this. For those readers familiar with the notion of the connection on the bundle of affine frames (as explained, for example, in the book by Kobayashi and Nomizu (1963)), take a 
closed loop which starts in simplex 1 and crosses once into simplex 2 and back. Then the affine holonomy of this loop is a translation in the tangent space of simplex 1 of magnitude

$$
x_{1}-P x_{2}
$$

where $x_{1}$ is the displacement vector in tangent space 1 between the two points where the curve crosses the boundary between the simplexes (its orientation, or sign, is a matter of definition-this is not important here), $x_{2}$ is the corresponding displacement vector in tangent space 2 and $P$ is the parallel transport from simplex 2 to 1 . If $P$ is the Christoffel choice then the vector of expression (1) vanishes, and likewise if it vanishes for every such loop, then the connection is the Christoffel one. Since the rotational holonomy is zero (so that, in any case, there is no ordinary curvature at the interface), a non-zero translation vector signals the presence of torsion.

The other way of seeing the presence of torsion, without needing the notion of the affine holonomy, is to integrate the torsion form directly. This is a little hard to interpret in general, but notice that, in the smooth case, if the curvature is zero, there exists an orthonormal frame obtained by parallelly transporting a particular frame at one point to all other points, in which the connection form is zero. In this frame the formula for the torsion 2-form is

$$
\tau^{A}=\mathrm{d} e^{A}
$$

where $e^{A}$ is the $R^{N}$-valued 1-form which defines the orthonormal frame.

We take this formula over to our situation, which differs in that the parallel frame $e^{A}$ may not be continuous. Then we integrate this expression on a 2 -surface which is bounded by the same type of loop as before, i.e. crossing into simplex 2 and back, and we get

$$
x_{1}^{A}-x_{2}^{A}
$$

where the vectors are now expressed in this special frame. This is not necessarily zero because, unless we choose the Christoffel connection, a particular displacement vector on the common face has different expressions when considered as belonging to the two different simplexes, and when compared by parallel transport. It is precisely this difference that the torsion is measuring. If formula (3) is written in a frame-independent way, and expressed as a tangent vector to simplex 1 , we recover formula (1).

\subsection{Simplicial manifold}

We consider a simplicial manifold $M$ of dimension $N$ made by glueing together many metric simplexes with the Christoffel connection. Since every subset consisting of a pair of $N$-simplexes is flat, the submanifold $M-M_{N-2}$ made by removing the ( $N-$ 2)-skeleton $M_{N-2}$ (the union of the triangles in four dimensions: more generally, the union of hinges) is flat (and hence smooth) because every point in $M-M_{N-2}$ has a neighbourhood entirely contained in two $N$-simplexes. Hence we can use all the usual notions of Riemannian geometry for $M-M_{N-2}$.

We investigate the geometry of the points of $M_{N-2}$, which we refer to as the singular points, and define geometrical objects at these points, entirely by referring to the geometry of the smooth $M-M_{N-2}$ which surrounds them.

\subsection{Tensors at a singular point}

It is possible to define a restricted notion of vectors and tensors at a singular point. For vectors, these turn out to be just the vectors parallel to the singularity, in the case 
when the torsion vanishes (but the curvature does not), as one might expect, but for tensors the situation requires a more formal treatment.

For a singular point $p$, there is a unique simplex $\sigma$ for which $p$ is an interior point. (For the exceptional case of a 0 -simplex, its geometrical point is defined to be interior.) For any simplex $a$ which contains simplex $b$ as a face (including $a$ itself) we use the notation $a>b$. We define the star of $p$ to be the union of the interiors of all simplexes $\Sigma$ for which $\Sigma>\sigma$ (see Maunder 1980). This is an open submanifold of $M$.

We define the tangent space at $p, T(p)$, as the space of parallel vector fields (i.e. whose covariant derivative vanishes) on the smooth manifold, $\operatorname{smooth}(p)$, consisting of $\operatorname{star}(p)$ with the singular points removed. This definition automatically gives an identification of $T(p)$ with a subspace of the tangent space $T(\Sigma)$ of any $N$-simplex $\Sigma>\sigma$. These vectors in $T(\Sigma)$ can also be characterised as the ones which are invariant under the action of the holonomy group of $\operatorname{smooth}(p)$. Clearly for different points $p$ in the interior of the same simplex $\sigma$, we may identify the tangent spaces $T(p)$. Thus we may talk of $T(\sigma)$.

Now in general the nature of the space $T(\sigma)$ depends on the curvature in $\operatorname{star}(p)$. Let us take the case of a singularity which is an ordinary cone, i.e. where $\sigma$ is a hinge. If the torsion is zero, the single holonomy element, for a loop encircling the singularity, is a rotation in the two-dimensional plane orthogonal to the hinge. Thus if the holonomy element is not the identity, $T(\sigma)$ is the $(N-2)$-dimensional tangent space to $\sigma$, with the metric of $\sigma$, and the obvious parallel connection with vectors in smooth $(\sigma)$. If the holonomy element is the identity, then $T(\sigma)$ is $N$ dimensional, in the same way at regular points. If the torsion is not zero, then $T(\sigma)$ may even be zero dimensional. However, we shall only consider the case of zero torsion in the following.

For simplexes of lower dimension than a hinge, where the conical structure of $\operatorname{star}(\sigma)$ is more complicated, one should note that the vectors parallel to $\sigma$ are always included in the tangent space $T(\sigma)$. This is because if we consider the corresponding vector in $T(\Sigma)$, for an $N$-simplex $\Sigma>\sigma$, such vectors lie parallel to every hinge $h>\sigma$, and so are invariant under holonomy elements for loops around all of the hinges $h$ in $\operatorname{star}(\sigma)$. Hence they are invariant under the whole holonomy group of $\operatorname{smooth}(\sigma)$ and can be extended to parallel vector fields throughout $\operatorname{smooth}(\sigma)$.

To define the tangent space in this way, on its own, is perhaps a bit fussy. But the real point is that we make a similar definition for tensors defined at (interior points of) a simplex $\sigma$. The space of tensors of type $(r, s)$ at $\sigma$ is defined to be the space of parallel tensor fields on $\operatorname{smooth}(\sigma)$. Note that these are not the $(r, s)$ tensor powers of the tangent space $T(\sigma)$. For example, in four dimensions, the space of antisymmetric $(4,0)$ tensors at a hinge $\sigma$ of non-zero curvature is one dimensional, spanned by the usual $\varepsilon_{A B C D}$. The space of antisymmetric $(2,0)$ tensors is two dimensional, spanned by $U_{A B}$ and $\frac{1}{2} \varepsilon_{A B C D} U^{C D}$, where $U_{A B}=V_{A} W_{B}-W_{A} V_{B}$, and $V$ and $W$ span the twodimensional $T(\sigma)$.

\section{The energy-momentum}

\subsection{Distributions versus the piecewise integral method}

There are two ways of presenting geometrical quantities on a Regge manifold. The first is to use tensor-valued distributions directly. This technique is not followed in this paper because it involves introducing some technical detail concerning the test function spaces which is not necessary for an understanding of the results of $\S \S 4$ and 
5. Instead, we use a second technique, which is to define directly a tensor for every given surface in $M$ of a suitable class of surfaces. These tensors are the tensors that would result if one formulated the theory in terms of distributions and then integrated the distribution on the given surface.

This will become clearer by considering the particular case we have in mind, namely the definition of the energy-momentum.

\subsection{The definition of the Einstein term}

The class of surfaces we have in mind are $(N-1)$-dimensional orientable surfaces which lie entirely within (the closure of) the star of one particular hinge $h$. Furthermore, we require that they intersect all simplexes in 'general position', i.e. in a $k$-dimensional surface for a $(k+1)$-simplex (and not at all for a 0 -simplex).

For each such surface $C$, together with a choice $n$ of one of the two normal directions to $C$, we define a vector in $T(h)$ as follows:

$$
p^{A}(C, n)=\delta_{h} \int_{C \cap h} m^{A} \Omega \text {. }
$$

Some of the terms used have to be explained. The deficit angle of $h$ is denoted $\delta_{h}, \Omega$ is the usual volume measure for the $(N-3)$-dimensional surface of integration, $C \cap h$. The vector $m^{A}$ is the unit normal to $C \cap h$ as embedded in $h$, with its direction determined to agree with the direction of $n$. The integral over vectors is well defined because all the vectors lie in the same tangent space. Now it is clear that the definition cannot be extended to surfaces $C$ which intersect more than one hinge, since one would have to sum over vectors in different tangent spaces on a curved manifold.

The interpretation of $p$ is that it represents the energy-momentum flow through surface $C$ in the direction represented by $n$. If we have some more general $(N-$ 1)-dimensional surface $H$ which intersects simplexes in 'general position' but is not restricted to the star of one hinge, together with some normal orientation of the surface $n$, then we can consider a splitting of $H$ into a set of cells $\{C(h)\}$, each of which intersects only one hinge $h$, and such that each hinge $h$ intersects only one cell, $C(h)$. Then the set of vectors $\left\{p^{A}(C(h), n): h\right.$ intersecting $\left.H\right\}$ represents the energymomentum flow through $H$. In general relativity, with energy-momentum tensor $T^{A B}$, we say that $T^{A B} n_{B}$ represents the energy-momentum flow through a hypersurface $H^{\prime}$ with normal $n_{B}$. The quantities $p$ are the discrete analogues of this vector field on $H^{\prime}$. In neither case can one form a sum of total energy-momentum, at least without introducing some other vector or spinor field.

Some justification should be provided for this particular definition. The first point is that the results of this paper show that it is a useful definition to make because the quantities can be related directly to the Regge equations of motion. Thus it gives a geometrical interpretation of the Regge equations of motion. Secondly, I should like to make a conjecture that, for some suitable concrete formulation of the problem, if a piecewise-flat metric approximates a smooth one, then the energy-momentum flow of the piecewise-flat metric through some suitable hypersurface, as defined here, approximates the energy-momentum flow of the smooth metric.

A third rationalisation for making this definition is that if one forms the distributional analogue of the Riemann curvature tensor, as considered by Regge, and calculates the Einstein tensor using the same formula as in the smooth case, one arrives at the above formulation (Barrett 1986, Miller 1986). Thus if one considers a piecewise-flat 
manifold as an ordinary spacetime with distributional matter in it where the hinges are, one is recording precisely the matter energy-momentum flow.

In coordinates, one can write the energy-momentum distribution in the following way. If one has $x^{1}, x^{2}, \ldots, x^{N}$ as coordinates for an $N$-simplex $\Sigma>h$ such that the metric of $\Sigma$ in these coordinates is the Kronecker delta $\delta_{A B}$, and $h$ lies in the coordinate surface defined by $x^{1}=x^{2}=0$, then as a tensor in $\Sigma$, the distribution for the energymomentum corresponding to hinge $h$ is

$$
\gamma^{A B}=\delta_{h} \delta\left(x^{1}\right) \delta\left(x^{2}\right) k^{A B}
$$

where the tensor $k^{A B}$ is defined by $k^{A B} v_{B}=v^{A}$ for $v^{A}$ parallel to $h, k^{A B} v_{B}=0$ for $v^{A}$ orthogonal to $h$. It is the metric restricted to $h$. The last two $\delta$ denote Dirac delta functions. One can show that

$$
p^{A}=\int_{C} \gamma^{A B} n_{B} \Omega^{\prime}
$$

(where $\Omega^{\prime}$ is the volume measure for $C$ and $n^{A}$ the normal to $C$ (in $\Sigma$ )) is equivalent to the definition (4). These coordinate expressions are a little tricky and the geometrical definition (4) is to be preferred. One should note that, should one develop the distributional aspect of the definition of energy-momentum further, there is no contribution from simplexes of dimension other than $N-2$, except in the case considered below, when one has a cosmological constant. This is a matter of definition.

\subsection{The cosmological term}

In general relativity, when one has a cosmological term in the equations of motion, the energy-momentum has two terms: the Einstein tensor $G^{A B}$ and the cosmological term $\lambda g^{A B}$ :

$$
T^{A B}=G^{A B}+\lambda g^{A B}
$$

We define a cosmological term in the energy-momentum flow through a surface in Regge calculus in a similar manner to the Einstein term considered above. We consider a different class of surfaces, however, namely those $(N-1)$-dimensional orientable surfaces which lie entirely within one $N$-simplex $\sigma$. The contribution to the energy-momentum flow through surface $C$, with normal $n^{A}$, is

$$
p^{A}=\lambda \int_{C} \Omega^{\prime} n^{A}
$$

where, as before, $\Omega^{\prime}$ is the volume measure of $C$. One can justify this definition in the same way as for the Einstein term. The definition is clearly just equation (6) with $\gamma^{A B}=\lambda g^{A B}$, instead of (5), which represents the Einstein term.

Thus for a theory with cosmological constant $\lambda$, the energy-momentum flow through surface $H$ consists of a set of vectors in the tangent spaces of the hinges intersecting $H$, representing the Einstein term, together with a set of vectors in the tangent spaces of the $N$-simplexes, representing the cosmological term. This flow is related to the Regge equations of motion with cosmological constant $\lambda$ in the next sections.

Similarly, I conjecture that, for an approximating smooth manifold, the flow approximates the continuum energy-momentum flow as calculated from equation (7). 


\section{The resolution theorem}

The previous section defined the energy-momentum flow (4) due to the Einstein term as the deficit angle times an integral of the normal to a $(N-3)$-dimensional hypersurface in an $(N-2)$-dimensional simplex. Similarly, the cosmological term $(8)$ is the integral of the normal to an $(N-1)$-dimensional hypersurface in an $N$-simplex. We compare these with the Regge equations. The letter $k$ is used to denote an edge and the notation $|\sigma|$ denotes the volume of a general simplex $\sigma$. Thus $|k|$ is the length of $k$. $S$ denotes the Regge action and $V$ the volume of the manifold. The variation of the Regge action is, as shown by Regge, a sum over hinges $h$

$$
\frac{\partial S}{\partial|k|}=\sum_{h>k} \delta_{h} \frac{\partial|h|}{\partial|k|}
$$

and the variation of the cosmological term, the volume of the manifold, is a sum over $N$-simplexes $\sigma$

$$
\frac{\partial V}{\partial|k|}=\sum_{\sigma>k} \frac{\partial|\sigma|}{\partial|k|} .
$$

Putting these four results together, it seems that one should investigate whether there is a relation between the variation of a volume of a simplex and the integral of the normal to a hypersurface of one lower dimension passing through the simplex in some manner. There is indeed such a relation if one picks some reasonably special hypersurface. It is the aim of this section to present this result.

Let $\sigma$ be an arbitrary $N$-dimensional metric simplex. First, it is worthwhile recording the formula for the variation of its volume

$$
\frac{\partial|\sigma|}{\partial|k|}=\frac{|k|}{N(N-1)} \cot (f, g)|f \cap g| .
$$

In this formula, $f$ and $g$ are the two $N-1$ faces given by deleting one of the vertices of edge $k$ from $\sigma$. The angle $(f, g)$ is the angle between these faces, where they hinge at the $N-2$ face $f \cap g$, the face opposite $k$. Clearly the formula is a direct generalisation of Regge's for $N=2$. The proof of this formula will not be given, as it is not used directly in the following.

Of much more central interest is the next result. Let $v$ be the vertex of $\sigma$ and $f$ the opposite $N-1$ face, i.e. the face not containing $v$. Then let $n(f)$ be the vector with length $|f|$ and directed along the outward normal to $f$ (i.e. the integral of the normal to $f$ ) and $u(k)$ the unit vector pointing away from $v$ and along an edge $k$ which contains $v$.

\section{Theorem.}

$$
n(f)=N \sum_{k>\nu} \frac{\partial|\sigma|}{\partial|k|} u(k)
$$

Before giving the proof of this formula, its use is discussed. If $W$ is a hypersurface in $\sigma$ which cuts $\sigma$ into iwo pieces, one piece containing $v$ and the other piece containing $f$, and is such that the integral of its normal $n(W)$ is $1 / N$ times $n(f)$, then $n(W)$ is the sum over precisely those edges which intersect $W$, of vectors pointing along those edges, with magnitude equal to the variation of the volume with respect to that edge 
length. For example, such a surface $W$ can be obtained by sliding the face $f$ down $\sigma$ a suitable distance towards the vertex $v$. (This is not, however, the canonical example which plays a role in the following.) There are more general surfaces which have this 'resolution' property. As shall be shown below, for every partition of the vertices of $\sigma$ into two sets, there is a surface $W$ dissecting $\sigma$ into two pieces and separating the two sets of vertices, such that

$$
n(W)=\sum_{k \in \operatorname{int}(W)} \frac{\partial|\sigma|}{\partial|k|} u(k)
$$

where the sum is over int $(W)$, the set of edges which intersect $W$, and where the unit vectors $u(k)$ all point along the edges, away from $W$ in the same sense as the normal.

In the context of a manifold, these special hypersurfaces, to be described fully below, which have property (12) can be fitted together to form a hypersurface through the manifold, the resolution hypersurface $H$. The way in which they fit together is described in $\S 5$. This means that, on such a hypersurface, we can regroup the 'contributions' to the energy-momentum flow:

$$
\left\{\delta_{h} n(H \cap h): h \in \operatorname{simplex}(N-2)\right\} \quad \text { and } \quad\{\lambda n(H \cap \sigma): \sigma \in \operatorname{simplex}(N)\}
$$

(simplex $(n)$ denotes the set of $n$-simplexes) into a set

$$
\left\{\left(\sum_{h>k} \frac{\partial|h|}{\partial|k|} \delta_{h}+\sum_{\sigma>k} \frac{\partial|\sigma|}{\partial|k|} \lambda\right) u(k): k \in \operatorname{int}(H)\right\}
$$

by resolving the normals into vectors defined on the edges, by (12), and then summing the contributions to the energy-momentum at each edge. One should notice that this operation is well defined because all the vectors $u(k)$ are in the tangent space to the edge $k$.

Written in this way, the set of vectors in (13), labelled by $k$, are clearly just $u(k)$ times (9) and $\lambda$ times (10): in other words, the left-hand side of the Regge equations of motion with cosmological constant $\lambda$. This gives a direct link between the distributional energy-momentum and the discrete variation of the Regge action.

\subsection{Details of the resolution theorem}

Again, let $\sigma$ be an arbitrary $N$-dimensional simplex. For any partition of the vertices of $\sigma$ into two sets, $S$ and $S^{*}$, we define a hypersurface $W(S)$ as a certain subcomplex of the derived complex $\sigma^{\prime}$ of $\sigma$. We shall also denote the faces spanned by $S$ and $S^{*}$ by the same letters. Recall (Maunder 1980, p 49) that the derived complex is defined by adding a vertex at the barycentre of each face of $\sigma$, and that the $I$-simplexes of $\sigma^{\prime}$ are the sets

$$
\left(\hat{\sigma}_{0}, \hat{\sigma}_{1}, \ldots, \hat{\sigma}_{l}\right)
$$

where $\hat{\sigma}_{X}$ is the centre of face $\sigma_{X}$ (not necessarily $X$ dimensional) and $\sigma_{0}<\sigma_{1}<\ldots<\sigma_{I}$. $W(\sigma, S)$ is defined as the polyhedron of the subcomplex $K(\sigma, S)$, which consists of the simplexes for which $\sigma_{0}$ is not contained in the faces $S$ or $S^{*}$. W(S) does not contain any points of $S$ or $S^{*}$. One should note that we include the case where the set of vertices $S$ or $S^{*}$ is the empty set. The face with no vertices, a -1 -simplex, is defined to be the empty set. From the definitions, in this case, $K(S)$ and $W(S)$ are likewise empty. 
First it is shown that $W(S)$ is a manifold and that it cuts $\sigma$ into two pieces, one containing $S$ and one containing $S^{*}$. We define two closed subsets of $\sigma, F(S)$ and $F\left(S^{*}\right), F(S)$ being the polyhedron of the subcomplex of $\sigma^{\prime}$ consisting of the simplexes for which $\sigma_{0}$ is not contained in $S^{*}$, and the other way around for $F\left(S^{*}\right), \sigma_{0}$ not being contained in $S$. Clearly $S$ is in $F(S), S^{*}$ in $F\left(S^{*}\right)$ and $W(S)$ is the intersection of $F(S)$ and $F\left(S^{*}\right)$, thus cutting $\sigma$ into the two pieces. To show that $W(S)$ is a manifold, it is possible to show that it is homeomorphic to $S \times S^{*}$. Notice that a point $p \in W(S)$ can be mapped to $S \times S^{*}$ by considering the projection by the unique straight line in $\sigma$ which intersects $S$ and $S^{*}$, as faces of $\sigma$, and passes through $p$. That is, $p \rightarrow(v, w)$, where

$$
p=\lambda v+(1-\lambda) w \quad v \in S, w \in S^{*} .
$$

The vertices of $K(S)$ are mapped to the points $(v, w) \in S \times S^{*}$, with $v$ a vertex of $S^{\prime}$ and $w$ a vertex of $S^{* \prime}$. This projection is continuous and non-linear. It is onto, because a line in $\sigma$ intersecting $S$ and $S^{*}$ must cross $W(S)$. It is into $(1-1)$ because $\lambda$ is determined uniquely by the following equation (14)†. Denoting the vertices of $S$ by $s\left(S^{*}\right.$ by $\left.s^{*}\right)$ and expressing $v, w$ in barycentric coordinates $\{x(s)\},\left\{y\left(s^{*}\right)\right\}, x, y \in[0,1]$, i.e.

$$
\begin{array}{ll}
v=\sum_{s \in S} x(s) s & w=\sum_{s^{*} \in S^{*}} y\left(s^{*}\right) s^{*} \\
\sum x(s)=1 & \sum y\left(s^{*}\right)=1
\end{array}
$$

then

$$
\lambda \max \{x(s)\}=(1-\lambda) \max \left\{y\left(s^{*}\right)\right\} .
$$

One can also see from (14) that the inverse map is continuous.

Now we come to the proof of (11). We regard the simplex $\sigma$ as a subset of $R^{N}$ with a fixed metric inner product, denoted by a dot, and start with the formula

$$
|\sigma|=(1 / N) n(f) \cdot(a-V)
$$

for the volume of $\sigma$, where the symbol $V$ has been used for the position vector of vertex $v$ and $a$ is an arbitrary point in the face $f$. Formula (15) is differentiated with respect to $V$, keeping the positions of the other vertices fixed. However, we regard $|\sigma|$ as a function of the edge lengths $|k|$ for the 1 -simplexes $k<\sigma$ and use the chain rule

$$
\frac{1}{N} n(f)=-\frac{\partial|\sigma|}{\partial V}=\sum_{k>v} \frac{\partial|\sigma|}{\partial|k|} u(k)
$$

which is the result (11).

With this intermediate result, the proof of the resolution theorem is easy. The precise statement of it is as follows.

Theorem. For any face $S$ of $\sigma$, formula (12) holds for the special surfaces $W(S)$ defined above. 
First, the result is proved for the case when $S$ is a single vertex $v$. In this case, we notice that the (geometric) boundary of $F(S)$ consists of the surface $W(S)$ and certain parts of the $N-1$ faces of $\sigma$ which contain $v$. Stokes theorem is used to calculate the integral of the normal to $W(S)$ as minus the integral of the normal on the other parts of the boundary of $F(S)$. An easy calculation shows that, on a particular $(N-1)$ face $g$ containing $v$, the boundary of $F(S)$ covers $1 / N$ times the volume of $g$. Adding these up over all the faces containing $v$ and using Stokes theorem again, we arrive at the conclusion that $n(W)$ is equal to $1 / N$ times the normal to the other remaining face, i.e. $f$. Then, using formula (11), the desired result is proved.

To treat the case where $S$ is a general set of vertices, we note that $F(S)$ is the union of $F(\{v\})$ for vertices $v \in S$ and that $n(W(S))$ is the sum of the $n(W(\{v\}))$ for $v \in S$. Using the resolution formula just proved for each of the $n(W(\{v\}))$, we note that the resolved components along the edges internal to the face $S$ cancel, leaving precisely the terms in (12).

\section{The resolution hypersurfaces}

In the previous section, a range of hypersurfaces which cut through a single simplex in different ways was discussed. This section presents a discussion of the way in which these hypersurfaces can be made to fit together, when the simplexes fit together to form a manifold.

Given a complex $C$ whose polyhedron is a manifold (we ignore the boundary, so this is taken as a manifold without boundary), a partition of the complex $C$ is defined as a partition of the vertices of each simplex of $C$ into two mutually exclusive sets in such a way that, if a simplex $f$ is a face of another simplex $\sigma$, the partition of the vertices of $f$ is just the restriction of the partition of $\sigma$. Such a partition of $C$ defines a subcomplex $K$ as the union, over all simplexes $\sigma$, of the subcomplexes $K(\sigma, S)$ defined in $\$ 4, S$ being defined by the partition. The polyhedron of $K$, denoted $H$, is the resolution hypersurface through $M$.

One of the main problems is to show that $H$ is a manifold. The way this is done is to provide an explicit mapping of the simplicial neighbourhood of each vertex in $K$ to a complex which is known to be an $N-1$ disc. Specifically, if $\hat{\sigma}_{M}$ is the centre of a $M$-simplex $\sigma_{M}$ (in this section the subscript labelling a simplex is equal to its dimension), and it lies in $H$, then the simplicial neighbourhood in $K$ of $\hat{\sigma}_{M}$ is isomorphic to the join of $W\left(\sigma_{M}, S\right)$ (where $S$ is one of the faces of $\sigma_{M}$ given by the partition) and the derived complex of the link $\dagger$ of $\sigma_{M}, L^{\prime}\left(\sigma_{M}\right)$. This is easily seen from the definition of the $(N-1)$-simplexes in the simplicial neighbourhood of $\hat{\sigma}_{M}$. These are

$$
\left(\hat{\sigma}_{N}, \hat{\sigma}_{N-1}, \ldots, \hat{\sigma}_{M}, \ldots, \hat{\sigma}_{1}\right)
$$

where $\sigma_{N}>\sigma_{N-1}>\ldots>\sigma_{M}>\ldots \sigma_{1}$. The simplex $\sigma_{1}$ is not contained in $S$ or $S^{*}$. In this formula $\sigma_{M}$ is fixed but the other simplexes can vary within the constraints. Consequently the simplicial neighbourhood is the join of the two complexes, of

$\dagger$ The simplicial neighbourhood of $\sigma$ is defined to be the subcomplex containing all simplexes $\Sigma>\sigma$, together with their faces. The link of $\sigma$ is then the subcomplex of the simplicial neighbourhood consisting of the simplexes having no vertex in common with $\sigma$. The link 'encircles' $\sigma$. For more details and some diagrams, see Maunder (1980). 
dimension $M-1$ and $N-M-1$, generated by their largest-dimensional simplexes

$$
\left(\hat{\sigma}_{M}, \hat{\sigma}_{M-1}, \ldots, \hat{\sigma}_{1}\right)
$$

and

$$
\left(\hat{\sigma}_{N}, \hat{\sigma}_{N-1}, \ldots, \hat{\sigma}_{M+1}\right) .
$$

The first is $W\left(\sigma_{M}, S\right)$ and the second can easily be seen to be simplicially homeomorphic to $L^{\prime}\left(\sigma_{M}\right) . L^{\prime}\left(\sigma_{M}\right)$ is empty if $N=M$.

Now $W\left(\sigma_{M}, S\right)$ is topologically an $M-1$ disc and $L^{\prime}\left(\sigma_{M}\right)$ an $N-M-1$ sphere (Rourke and Sanderson 1972) and so the join is an $N-1$ disc. This proves that $H$ is a manifold.

The converse is also true: if $H$ is an $(N-1)$-dimensional hypersurface in $M$ composed of a union over $\sigma$ of subcomplexes $W(\sigma, S)$, then this determines a partition of $K$. One should note that there will be simplexes $\sigma$ which do not intersect $H$ at all. In this case the partition of the vertices of $\sigma$ is into one set containing all the vertices and a second set containing none.

\section{Acknowledgments}

I should like to thank the following: $\mathrm{R}$ Sorkin and $\mathrm{M}$ Zähringer for discussing the convergence problem with me and I $\mathrm{T}$ Drummond for discussing the first-order formulation of Regge calculus and supplying me with a draft paper. A Royal Society European Science Exchange Programme fellowship, the Schweizeriche Nationalfonds and the Tomalla Foundation provided financial support.

\section{References}

Barrett J W $1985 P h D$ Thesis University of London 1986 Class. Quantum Grav. 3 203-6

Cheeger J, Mueller W and Schrader R 1984 Commun. Math. Phys. 92 405-54

Drummond I T 1986 Nucl. Phys. B 273 125-36

Feinberg G, Friedberg R, Lee T D and Ren H C 1984 Nucl. Phys. B 245 343-68

Hartle J B 1985 J. Math. Phys. 26 804-14

Kobayashi S and Nomizu K 1963 Foundations of Differential Geometry vol 1 (New York: Interscience)

Maunder C R F 1980 Algebraic Topology (Cambridge: Cambridge University Press)

Miller W A 1986 Found. Phys. 16143

Regge T 1961 Nuovo Cimento 19558

Rourke C P and Sanderson B J 1972 Introduction to Piecewise-Linear Topology (Berlin: Springer) 